03

\title{
Влияние локализованного импульсно-периодического подвода энергии на сверхзвуковое смешение \\ в каналах
}

\author{
(C) А.А. Желтоводов, Е.А. Пимонов
}

Институт теоретической и прикладной механики им. С.А. Христиановича СО РАН, Новосибирск

E-mail: zhelt@itam.nsc.ru

Поступило в Редакцию 31 января 2017 г.

На основе численного моделирования сверхзвуковых течений в плоских каналах с сужающимися и расширяющимися входными участками обосновывается возможность интенсификации смешения сверхзвуковой газовой струи пониженной плотности с окружающим спутным сверхзвуковым потоком с помощью локализованного импульсно-периодического подвода энергии. Демонстрируется влияние положения струи и энергоисточника на формирование крупномасштабных вихревых структур, сопутствующих интенсификации процесса смешения.

DOI: 10.21883/PJTF.2017.16.44929.16723

Поиск эффективных способов интенсификации смешения сверхзвуковых газовых потоков остается одной из актуальных задач современной аэрогазодинамики. В значительной степени это обусловлено разработкой гиперзвукового прямоточного воздушно-реактивного двигателя (ГПВРД) для реализации эффективного горения струй подаваемого топлива (например, водорода) в окружающем сверхзвуковом воздушном потоке в камере сгорания. Исторический обзор выполненных обширных исследований интенсификации смешения применительно к ГПВРД с помощью различных механических устройств дан в работе [1]. Возможности улучшения смешения и эффективности горения путем предварительной подачи струи топлива в набегающий поток перед воздухозаборником или в изоляторе перед камерой сгорания анализировались в работах $[2,3]$. В качестве перспективного способа воздействия на эти процессы в настоящее время рассматривается 
подвод энергии с помощью оптических и электрических разрядов [4-6]. Как показано, например, в работах [7,8], локализованный импульснопериодический энергоподвод стимулирует образование крупномасштабных вихревых структур в распространяющихся в сверхзвуковом потоке спутных струях в условиях их взаимодействия с косым скачком уплотнения. Такие вихревые структуры возникают вследствие неустойчивости Рихтмайера-Мешкова при взаимодействии локальных зон энергоподвода со скачком и способствуют интенсификации смешения струй с окружающим потоком. Поскольку в тракте ГПВРД реализуется сложная система пересекающихся падающих и отраженных от внутренних поверхностей скачков уплотнения и волн разрежения, представляют интерес исследования сверхзвукового смешения в таких условиях.

В настоящей работе рассматривается воздействие локализованного импульсно-периодического подвода энергии на развитие двумерной сверхзвуковой спутной изобарической струи при ее взаимодействии с различными последовательностями пересекающихся скачков уплотнения и волн разрежения, возникающими в плоских каналах с сужающимися и расширяющимися участками. Как и в работах $[7,8]$, с этой целью проведены численные расчеты в рамках нестационарных уравнений Эйлера для идеального совершенного газа. При этом использовалась конечно-объемная схема типа схемы Годунова третьего порядка по пространству, в рамках которой потоки через грани расчетной ячейки на текущем временно́м слое находятся решением локальной одномерной задачи Римана HLLEM-методом. С целью повышения порядка точности по пространству без потери монотонности алгоритма применялась процедура MUSCL-интерполяции. Для расчета газодинамических величин на шагах по времени применялась явная схема Рунге-Кутты третьего порядка. Подвод энергии моделировался источниковым членом в уравнении сохранения энергии. Для расчета удельной мощности на единицу массы задавались энергия, подводимая за один импульс, начальная форма и размер источника энергии.

В качестве граничных условий на левой границе расчетной области, соответствующей входу в канал, задавались постоянные параметры в однородном сверхзвуковом потоке, а также распространяющейся в нем спутной струи. На правой границе (выходе из канала) использовались стандартные „мягкие“ условия, а на верхней и нижней поверхностях каналов - условия непротекания. Сходимость полученных решений проверялась предварительными расчетами на равномерных регулярных

Письма в ЖТФ, 2017, том 43, вып. 16 
сетках $(2400 \times 400,3000 \times 500$ и $3600 \times 600)$, что позволило обосновать приемлемость сетки с размерностью $3600 \times 600$.

В анализируемых случаях на входе в каналы задавался равномерный сверхзвуковой поток с числом Маха $M_{\infty}=3.0$, давлением торможения $p_{0}=0.25 \mathrm{MPa}$ и температурой торможения $T_{0}=288 \mathrm{~K}$. При этом плотность в изобарической спутной струе в исходном сечении была в 2 раза меньше, чем в окружающем потоке, а постоянное по ее толщине число Маха принималось равным $M_{j}=1.05$. Начальная толщина плоской струи на левой границе расчетной области задавалась равной $h_{j}=0.01 \mathrm{~m}$.

Полученное в расчетах поле градиентов плотности (рис 1,a) характеризует установившуюся в момент времени $t=12000 \mu$ s газодинамическую структуру течения в канале с сужающимся входным участком и расположенной в его плоскости симметрии $\left(y / h_{j}=10.5\right)$ двумерной струей в условиях без энергоподвода. Высота образованного параллельными горизонтальными поверхностями входного сечения канала $H_{1} / h_{j}=21$, угол отклонения симметричных верхней и нижней поверхностей сжатия $\alpha=10^{\circ}$, высота следующей части канала с параллельными поверхностями $H_{2} / h_{j}=17$. В рассматриваемых условиях струя проникает через распространяющиеся от поверхностей сжатия навстречу друг другу косые скачки уплотнения. При этом внутри струи в сечении $x / h_{j}=11$ формируется прямой скачок-предвестник, который вне струи трансформируется в наклонные скачки, достигающие параллельных стенок канала за поверхностями сжатия. За скачкомпредвестником на участке $10.7<x / h_{j} \leqslant 65$ в струе образуются две отчетливо выраженные утолщенные зоны дозвукового течения. Высота первой зоны постепенно растет, и в сечении $x / h_{j}=21.8$ становится максимальной. Распространяющиеся от поверхностей сжатия косые скачки уплотнения отражаются в этом сечении от границ струи веерами волн разрежения, которые способствуют ускорению течения и уменьшению высоты дозвуковой зоны. Под влиянием дополнительных волн разрежения, направленных к центру канала от концов поверхностей сжатия, струя разгоняется в сечении $x / h_{j} \approx 36.7$ до числа Маха на оси $M_{j}=0.72$ и достигает минимальной толщины. При отражении этих волн разрежения от струи образуются направленные к стенкам канала волны сжатия, объединяющиеся в слабый скачок.

Распространяющиеся от вершины дозвуковой зоны косые скачки уплотнения отражаются от стенок канала (рис. $1, a$ ). В результате их 


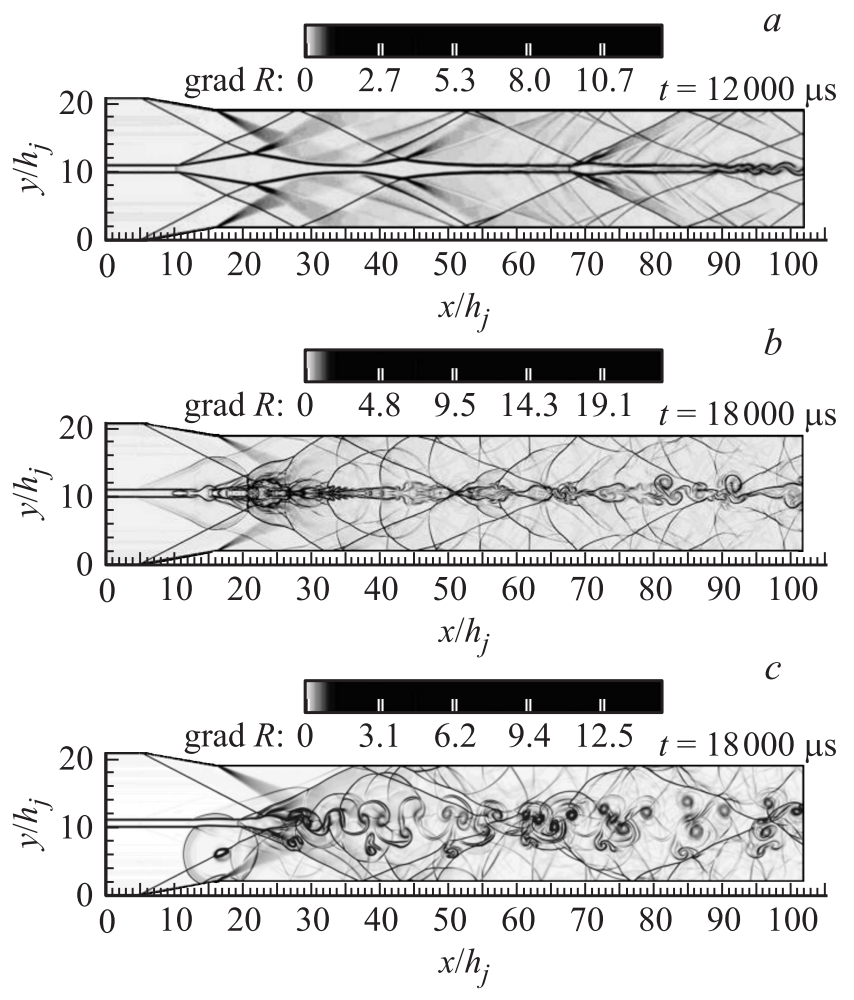

Рис. 1. Течение в сужающемся канале $\left(\alpha=10^{\circ}\right)$ со струей вдоль его плоскости симметрии: $a-$ без подвода энергии, $b-$ с пульсирующим источником энергии $(E=0.0512 \mathrm{~J}, f=5 \mathrm{kHz})$ в струе $\left(x_{0} / h_{j}=5, y_{0} / h_{j}=10.5\right), c-$ с пульсирующим энергоисточником под струей $\left(x_{0} / h_{j}=5, y_{0} / h_{j}=6\right)$.

взаимодействия со струей образуется вторая утолщенная дозвуковая зона. Ее начало примерно соответствует сечению $x / h_{j} \approx 36.7$. С учетом дозвуковой скорости в струе перед этой зоной не образуется прямой скачок, а во внешнем течении формируются лишь косые скачки. При $x / h_{j} \approx 65.2$ струя разгоняется до звуковой скорости. Наконец, распространяющиеся косые скачки от начала второй утолщающейся зоны, отражаясь от стенок канала, стимулируют образование третьей дозвуко-

Письма в ЖТФ, 2017, том 43, вып. 16 


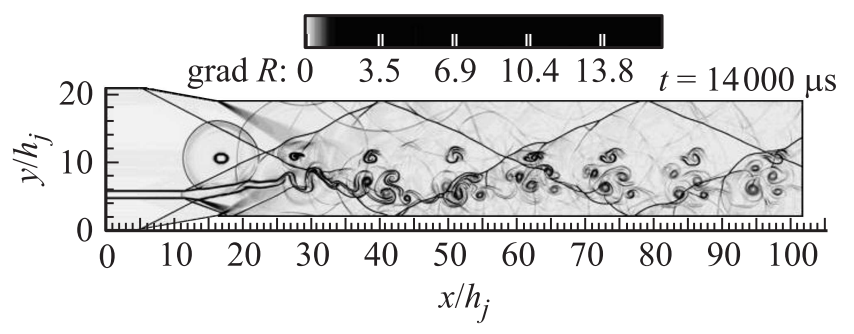

Рис. 2. Течение в сужающемся канале $\left(\alpha=10^{\circ}\right)$ со струей, расположенной ниже плоскости симметрии канала $\left(y / h_{j}=5.25\right)$, и с пульсирующим источником энергии в плоскости симметрии канала $\left(x_{0} / h_{j}=5, y_{0} / h_{j}=10.5\right)$.

вой зоны в струе при $67.7 \leqslant x / h_{j} \leqslant 87.5$, перед которой внутри струи наблюдается прямой скачок-предвестник. Число Маха непосредственно перед ним $M_{j} \approx 1.14$. Характер течения в струе, развивающейся в канале в условиях влияния падающих и отраженных скачков уплотнения и волн разрежения, не обнаруживает признаков ее активного перемешивания с окружающим сверхзвуковым потоком. Лишь при $x / h_{j}>88$ в струе появляются волны, обусловленные неустойчивостью Кельвина-Гельмгольца, которые при дальнейшем развитии могут постепенно трансформироваться в вихревые структуры, способствующие интенсификации смешения.

Для изучения влияния локализованного подвода энергии на развитие струи в канале в сформировавшееся течение (рис. 1,a) вводился пульсирующий с частотой $f=5 \mathrm{kHz}$ энергоисточник с эллипсоидальным сечением. Длины горизонтальной и вертикальной полуосей такого источника составляли соответственно $\Delta x / h_{j}=0.5$ и $\Delta y / h_{j}=0.25$. Подводимая в импульсе энергия была равна $E=0.0512 \mathrm{~J}$, а продолжительность импульса $\tau=10 \mathrm{~ns}$. Положение области энергоподвода задавалось координатами центра такого источника $x_{0}, y_{0}$. Полученные результаты в условиях импульсно-периодического подвода энергии на оси струи $\left(x_{0} / h_{j}=5, y_{0} / h_{j}=10.5\right)$ перед первой дозвуковой зоной (рис. $1, b$ ) демонстрируют возникающие вследствие неустойчивости Рихтмайера-Мешкова вихревые структуры непосредственно за пересекающимися косыми скачками, распространяющимися от поверхности сжатия. Размеры и темпы роста образующихся вихрей заметно меньше, 


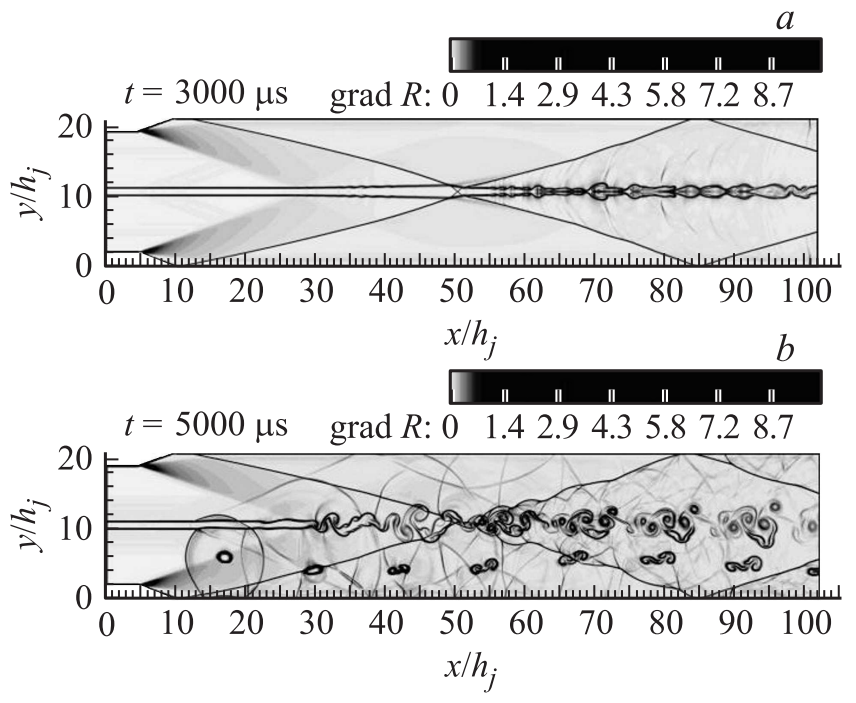

Рис. 3. Течение в расширяющемся канале $\left(\alpha=-20^{\circ}\right)$ со струей вдоль его плоскости симметрии: $a-$ без энергоподвода, $b-$ с пульсирующим энергоисточником $(E=0.0512 \mathrm{~J}, f=5 \mathrm{kHz})$ под струей $\left(x_{0} / h_{j}=5, y_{0} / h_{j}=6\right)$.

чем в случаях взаимодействия струи с косым скачком уплотнения и последующими волнами разрежения в окрестности наклонной ступеньки на нижней поверхности при отсутствии верхней стенки канала [7,8]. Как было показано в этих работах, при подводе энергии в набегающем потоке вне струи в таких условиях распространяющиеся от энергоисточника близкие к сферическим ударные волны инициируют в ней начальные возмущения, достаточные для формирования крупномасштабных вихревых структур. Согласно полученным таким же способом результатам, располагая энергоисточник под струей $\left(x_{0} / h_{j}=5, y_{0} / h_{j}=6\right)$, удается активизировать появление более крупных вихрей в канале (рис. 1,c). Качественно аналогичный характер течения, свидетельствующий о заметной интенсификации смешения, реализуется и в условиях энергоподвода на оси канала $\left(x_{0} / h_{j}=5, y_{0} / h_{j}=10.5\right)$ над струей, расположенной ближе к его нижней стенке (рис. 2).

Письма в ЖТФ, 2017, том 43, вып. 16 
Течение в канале с расширяющимся входным участком $\left(H_{1} / h_{j}=17\right.$, $\left.\alpha=-20^{\circ}, H_{2} / h_{j}=21\right)$ характеризуется последовательностью распространяющихся от вершин наклонных уступов пересекающихся волн разрежения, которые предшествуют образующейся за уступами системе пересекающихся скачков уплотнения, проникающих вниз по потоку (рис. 3,a). Струя, распространяющаяся в плоскости симметрии канала, предварительно ускоряется волнами разрежения и проникает через пересекающиеся скачки без признаков активного перемешивания с внешним потоком. Импульсно-периодический подвод энергии под струей $\left(x_{0} / h_{j}=5, y_{0} / h_{j}=6\right)$ в таких условиях способствует заметной интенсификации смешения (рис. $3, b$ ), как и в рассмотренных выше случаях.

Таким образом, выполненные исследования демонстрируют принципиальную возможность интенсификации процесса смешения сверхзвуковых струй с окружающим спутным сверхзвуковым потоком в каналах с помощью локализованного импульсно-периодического энергоподвода. Показано, что подвод энергии вне струи более эффективен для генерации в ней крупномасштабных вихревых структур, способствующих интенсификации процесса смешения, чем энергоподвод внутри струи. Планируемые дальнейшие экспериментальные и расчетные исследования позволят проанализировать влияние интенсивности и частоты энергоподвода на процесс смешения и развитие эффектов запирания течений в каналах различной конфигурации, а также уточнить влияние пространственных и вязких эффектов.

\section{Список литературы}

[1] Seiner J.M., Dash S.M., Kenzakowski D.C. // J. Propulsion Power. 2001. V. 17. N 6. P. 1273-1286.

[2] Vinogradov V.A., Shikhman Yu.M., Segal C. // Appl. Mech. Rev. Transact. of the ASME. 2007. V. 60. P. 139-148.

[3] Tuner J.C., Smart M.K. // AIAA J. 2010. V. 48. N 4. P. 829-838.

[4] Adelgren R.G., Elliott G.S., Crawford J.D., Carter C.D., Donbar J.M., Grosjean D.F. // AIAA J. 2005. V. 43. N 4. P. 776-791.

[5] Savelkin K.V., Yarantsev D.A., Adamovich I.V., Leonov S.B. // Combustion Flame. 2015. V. 162. P. 825-835. 
[6] Иванов В.В., Сквориов В.В., Стародубщев М.А., Чернышев С.Л. // Уч. зап. ЦАГИ. 2016. Т. XLVII. № 4. С. 3-11.

[7] Желтоводов А.А., Пимонов Е.А. // Письма в ЖТФ. 2013. Т. 39. В. 22. С. $73-$ 79.

[8] Zheltovodov A.A., Pimonov E.A. // Int. Conf. on the methods of aerophysical research: Abstracts. June 30-July 6, 2014. Novosibirsk, Russia, 2014. P I. P. 234235.

Письма в ЖТФ, 2017, том 43, вып. 16 